\title{
Guest Editorial: Special Issue on Emerging Trends in Modeling and Analysis of Complex Systems
}

\author{
Abhijin Adiga ${ }^{1}$ S. S. Ravi ${ }^{1} 2^{*}$ and Anil Vullikanti ${ }^{3}$
}

\section{Complex Systems, Network Dynamics and Graph Mining}

Across domains as diverse as public health, ecology, social science and critical infrastructure, a number of research questions are being studied from a complex systems perspective. Many such questions arise in the analysis of the spread of contagions such as infectious diseases and memes, natural disasters, failures in infrastructure networks, and biological processes in gene regulatory networks. Research on modeling and analyzing such phenomena has attracted a lot of attention in recent times, including several works that have emerged in the backdrop of the ongoing COVID-19 pandemic. This trend has been fueled by unprecedented growth in the volume and variety of available data coupled with developments in high performance computing and big data analytics. This special issue provides a peek into this emerging trend. The cover illustration by Henning Mortveit (Biocomplexity Institute and Initiative and Department of Engineering Systems and Environment, University of Virginia, USA), featured in the article by Bisset, Cadena, Khan and Kuhlman, illustrates the complex interactions of human beings among themselves as well as with built infrastructure and how such interactions can be modeled using networkbased dynamical systems. The cover illustration also captures the theme of many articles appearing in this special issue. These articles collectively address various topics in complex networked systems, including designing, understanding, and evaluating systems represented using networks and agent-based models, their theoretical foundations, and applications to various domains.

\section{Overview of Articles in the Special Issue}

The lead article in this special issue by Madhukara Putty provides a brief and informal overview of networked complex systems, their components, properties and applications. The article provides a number of examples of complex systems that occur in practice. Focusing on two specific examples, namely, epidemic propagation and transportation systems, the article discusses topics such as modeling complex systems and how simulation studies using such models provide insights to modelers as well as policy makers. This discussion highlights the challenges encountered in modeling and simulating networked complex systems. The article also points out that developments in machine learning and high performance computing will lead to significant advances in our ability to understand the behavior of complex systems.

The 12 main articles appearing in this special issue can be broadly divided into 3 categories. The focus of the first category is on infectious disease epidemiology. Not surprisingly, most of these articles apply the proposed techniques to the COVID-19 scenario. The second category comprises articles that focus on a variety of applications of dynamical systems and multiagent systems. These applications, which are different from infectious diseases, include disaster scenarios, memes and addictive behavior. The articles in the third category address topics in network science and graph mining with a variety of applications, from user behavior in Twitter to pangenomics. We now provide brief summaries of the main articles.

The articles in the first category cover various aspects of infectious disease spread, including theoretical frameworks, data integration, modeling, efficient implementation of epidemic simulations, and techniques for controlling epidemics. The scenarios considered span different spatial scales, and the models used differ widely in resolution and detail. The first article by Bisset, Cadena, Khan and Kuhlman provides a wellrounded overview of the agent-based modeling approach for computational epidemiology. The article first discusses the theoretical foundations of network-based models through the formalism of graph dynamical systems. This is followed by
${ }^{1}$ Biocomplexity Institute and Initiative, University of Virginia, Charlottesville, VA, USA.

${ }^{2}$ Department of Computer Science, University at Albany, State University of New York, Albany, NY, USA.

${ }^{3}$ Department of Computer Science, Biocomplexity Institute and Initiative, University of Virginia, Charlottesville, VA, USA. *ssravi@virginia.edu 
a description of contact network models of large populations that are induced by daily activities of individuals and the models for the propagation of epidemics over such networks. The authors also provide a good exposition of several methods for efficiently generating synthetic networks. In addition, they discuss how simulation results for various models can inform public policy.

The next two articles study infectious disease spread in crowded environments such as airports. Derjany, Namilae and Srinivasan model the infectious disease spread in pedestrian queues as an interaction between two component models, namely a force-based pedestrian dynamics model and an infectious disease spread model. This article contributes a novel methodology for parameter-space exploration of the proposed complex model to significantly reduce the number of simulations. Their model is applied to study the spread of diseases such as COVID-19 at airport security check points. Lahijani, Gayatri, Islam, Srinivasan, and Namilae consider a similar framework but focus on speeding up simulations on high performance computing systems equipped with graphics processing units (GPUs). A novel contribution of this article is a model (called the Constrained Linear Movement model) for pedestrian dynamics that has been specifically designed to perform efficiently on GPUs. Their architecture-aware approach optimizes the execution times of both individual simulation runs and experiments involving parameter sweeps.

The next article by Perumulla and Alam proposes a mesoscopic modeling approach for epidemiological simulations. Their method strikes a balance between high-resolution (but computationally intensive) modeling and aggregatelevel (but fast) modeling approaches. It has been designed to take advantage of accelerator-based architectures such as GPUs. The approach provides the capability to incorporate adaptive interventions by modifying the base simulation, thus allowing users to evaluate various counterfactual scenarios. The framework is applied to an Ebola spread model on a large population to illustrate the methodology and evaluate its performance.

Sinha, Nath, and Roy consider the spread and management of diseases such as COVID-19 that can cause both symptomatic and asymptomatic infections. It is important to consider asymptomatic carriers in the study of epidemics since such carriers have the potential to spread the disease to large number of individuals as they go about their daily activities. The authors use an individual-based Susceptible-Infected-Recovered-Deceased (SIRD) model along with ideas from game theory to study the problem. They also provide a thorough experimental evaluation of the efficacy of mass testing and precautionary non-pharmaceutical measures such as wearing masks, washing hands, and social distancing for mitigating the disease spread.

The second category of articles in this special issue focuses on other applications of agent-based models. Azizi, Mubayi and Mubayi use an agentbased model in conjunction with statistical techniques to study alcohol consumption behavior among students in a university setting. They consider student activities in different locations situated within a university campus and use survey data to design and calibrate their model. Through this model, they study the dynamics of alcohol drinking initiation and increased consumption. They also identify specific locations within a campus which are most influential in propagating alcohol-drinking behavior. Their results illustrate how agent-based models can be useful in developing long-term predictions regarding alcohol consumption among young students.

Parikh, Marathe and Swarup develop novel simulation analysis tools to study agent behavior in a complex multi-agent model of a nuclear disaster in an urban environment. The aim of their study is to provide behavior recommendations (e.g., seek immediate medical help, seek a shelter, try to reunite with family members) to agents. The premise is that the outcome of agent behaviors is context-based, and therefore, recommendations must account for an agent's situation. Their approach for developing recommendations first decomposes simulation outcomes into a causally relevant sequence of states and then uses techniques from reinforcement learning.

Rao and Krantz describe the theory of dynamical systems and its evolution from a framework used to study astronomy to new directions shaped by modern applications. Their discussion begins with an abstract dynamical system model over a topological space that arises from an ordinary differential equation (ODE) based model for population dynamics. They observe that the topological framework can be applied in both deterministic and stochastic contexts. They provide rigorous definitions of many associated concepts such as the phase space and trajectories of a dynamical system. The article points out how the abstract framework provides a useful generalization of various types of dynamical systems models studied in different disciplines such as physics and mathematics.

Bodas, Ganesh, and Manjunath study a setting with agents and heterogeneous servers, 
where each agent must choose a server. They point out that examples of such settings arise in several applications, including web server farms, transportation and healthcare. In such a setting, agents' behaviors can cause a congestion externality; that is, if too many agents choose the same server which provides the best service time, then the delay for each agent may increase. The authors model the setting as a congestion game in which choices made by self-interested agents can lead to equilibria that are sub-optimal with respect to social welfare (which can be thought of as a measure of the total cost incurred by the agents). The authors study the imposition of an admission price (which is similar to the notion of a Pigouvian tax used in Economics) to eliminate congestion externalities. The paper shows that there is an allocation of Pigouvian taxes so that the social welfare cost is minimized at an equilibrium (called a Wardrop equilibrium). The paper also presents rigorous results that characterize the structure of such equilibria.

The third category of articles focuses on network representations and graph mining which have many applications. Maurya and Ravindran study link prediction, the problem of predicting missing relationships in a network. This is a very well-studied problem in the conventional network setting, where the objective is to predict the existence of dyadic relationships (i.e., predicting an edge between two nodes of a network). The authors address the problem of link prediction in a hypergraph setting which allows non-dyadic relationships between entities. This topic has not received much attention in the literature. Maurya and Ravindran present a nice review of existing methods for this task. They use a tensor-based representation of hypergraphs to develop a novel link prediction algorithm based on tensor eigenvectors. They present a number of examples to illustrate their techniques.

The article by Saha and Ganguly considers temporal point processes (TPPs) which arise in a number of applications. Each TPP is a localized temporal sequence of discrete events. There are several application contexts where the TPPs influence each other. Examples include multiple Twitter hashtags seeking to gain user attention and products competing for attention in terms of user reviews in Ecommerce websites. The article provides a nice survey of the application domains where TPPs arise and techniques used in the literature to model TPPs. It also presents several novel extensions of these techniques to model competing TPPs. These extensions allow a modeler to optimize a variety of measures associated with the TPPs.

The article by Outten and Warren addresses pangenomics, a very active research topic under the general area of genomic analysis. In simple terms, a pangenome contains the entire gene set of all strains of a species. More specifically, it has a core genome that contains the genes present in all strains and a dispensable (or accessory) genome that has the genes present in some of the strains. The area of graphical pangenomics uses ideas from network science and data analytics to develop graphical models of pangenomes that can support a variety of tasks including sequence alignment, visualization, and functional genomics. Outten and Warren give a nice survey of the area, focusing on several recent developments. Many graph representation models are described based on concepts such as similarity context and regularized differences. The article covers stateof-the-art algorithms and software tools to carry out data analysis using various graph representations. It also discusses the capabilities that will be useful in new tools for graphical pangenomics.

\section{Final Remarks}

The articles in this special issue point out many important research directions in complex systems. Readers can get additional information regarding these directions and other research questions from the references cited in these articles. While the journal labels each published article as a "Review Article", readers will notice that many articles in this special issue not only review relevant related work, but also present new research results. We hope that these articles will enable interested researchers to obtain an understanding of some of the methods used in analyzing complex systems and to explore new directions.

\section{Publisher's Note}

Springer Nature remains neutral with regard to jurisdictional claims in published maps and institutional affiliations.

\section{Acknowledgements}

We express our sincere thanks to Professor G. K. Ananthasuresh (Editor-in-Chief) and Professor Aravind Srinivasan (Editorial Board Member) for inviting us to serve as guest editors for this special issue. We thank all the authors who contributed articles to this special issue. We also thank 
all the reviewers for carefully going through the submissions and providing very helpful suggestions to the authors. We are particularly thankful that these reviewers were able to provide excellent reviews within a short amount of time. We thank our colleague Professor Henning Mortveit for providing the cover image for this special issue. We received a great deal of help from Professor Ananthasuresh, the journal's staff members Ms. Kavitha Harish and Ms. M. V. Leelakshi, and Ms.
Sonal Choudhary of Springer. Their support and patience made it a pleasant experience for us to guest edit this special issue.

\section{Declarations}

\section{Conflict of interest}

The Guest Editors have no conflict of interest.

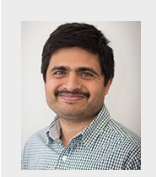

Abhijin Adiga is a Research Assistant Professor at the Network Systems Science and Advanced Computing (NSSAC) division, Biocomplexity Institute and Initiative, University of Virginia. Previously, he held positions as Research Assistant Professor, Senior Research Associate and Postdoctoral Associate at the Biocomplexity Institute of Virginia Tech. He received his $\mathrm{PhD}$ degree from the Department of Computer Science and Automation, Indian Institute of Science in 2011. His interests are in computational modeling, network science, algorithms, combinatorics and game theory. His current research focusses on the foundations of dynamical processes over networks and design and implementation of simulation systems to study invasive species spread, infectious diseases and socio-technical systems. He leads two projects funded by USAID and USDA on modeling invasive species to address food security challenges. His paper in AAAI'13 received "Honorable Mention For Outstanding Novelty of Research Question” award.

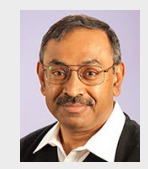

S. S. Ravi is a Research Professor in the Network Systems Science and Advanced Computing (NSSAC) Division of the Biocomplexity Institute and Initiative, University of Virginia, Charlottesville, VA, USA. He received his B.E. (Electrical Communication Engineering, 1977) and M.E. (Automation, 1979) degrees from the Indian Institute of Science and his Ph.D. in Computer Science in 1984 from the University of Pittsburgh,
USA. He was a member of the Computer Science faculty at the University at Albany-State University of New York from 1984 to 2016. From August 2016 to September 2018, he was with the Network Dynamics and Simulation Science Laboratory of the Biocomplexity Institute of Virginia Tech, Blacksburg, VA. His areas of interest include combinatorial algorithms, network science, discrete dynamical systems and data mining.

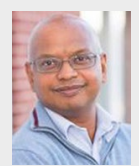

Anil Vullikanti is a Professor in the Department of Computer Science and at the Biocomplexity Institute and Initiative of the University of Virginia, Charlottesville, VA. Anil received his undergraduate degree from the Indian Institute of Technology,

Kanpur, and his Ph.D. from the Department of Computer Science and Automation at the Indian Institute of Science, Bangalore. He was a post-doctoral researcher at the MaxPlanck Institute for Informatics, a technical staff member at the Los Alamos National Laboratory and an Associate Professor in the Biocomplexity Institute and the Department of Computer Science at Virginia Tech, Blacksburg, VA. Anil's research interests are in the broad areas of approximation and randomized algorithms, dynamical systems, wireless networks, social networks, computational epidemiology and the modeling, simulation and analysis of socio-technical systems. He is the recipient of the NSF CAREER award and the DOE Early Career award. He is also an ACM Distinguished Member. 